POS $\quad \begin{aligned} & \text { PROCEEDINGS } \\ & \text { OF SCIENCE }\end{aligned}$

\title{
Mini-Superspace Quantum Supergravity and its Hidden Hyperbolic Kac-Moody Structures
}

\section{Philippe Spindel*}

Mons University, 20 place du Parc, 7000 Mons, Belgium

E-mail: philippe.spindeleumons.ac.be

\section{Thibault Damour}

Institut des Hautes Études Scientifiques, 91440 Bures-sur-Yvette, France

E-mail: damouraihes.fr

We provide a summary and references on our recent works on the complete quantisation of the Bianchi IX cosmological model in the framework of the minisuperspace.

Proceedings of the Corfu Summer Institute 2014 "School and Workshops on Elementary Particle Physics and Gravity",

3-21 September 2014

Corfu, Greece

${ }^{*}$ Speaker. 


\section{In brief}

The quantum dynamics of a supersymmetric squashed three-sphere has been completely solved. To this aim we have performed a dimensional reduction to one time-like dimension of the action of $\mathrm{D}=4$ simple supergravity for an SU(2)-homogeneous (Bianchi IX) cosmological model and imposed an appropriate choice of the gravitational gauge. The quantisation of the homogeneous gravitino field leads to a 64-dimensional fermionic Hilbert space. After imposition of the diffeomorphism constraints, the wave function of the Universe becomes a 64-component spinor of Spin $(8,4)$ depending on the three squashing parameters, which satisfies Dirac-like, and Klein-Gordon-like, wave equations describing the propagation of a "quantum spinning particle" reflecting off spindependent potential walls. The algebra of the supersymmetry constraints and of the Hamiltonian one is found to close. One finds that the quantum Hamiltonian is built from operators that generate a 64-dimensional representation of the (infinite-dimensional) maximally compact sub-algebra of the rank-3 hyperbolic Kac-Moody algebra $A E_{3}$. The (quartic-in-fermions) squared-mass term $\widehat{\mu}^{2}$ entering the Klein-Gordon-like equation has several remarkable properties: (i) it commutes with all the other (Kac-Moody-related) building blocks of the Hamiltonian; (ii) it is a quadratic function of the fermion number $N_{F}$; and (iii) it is negative in most of the Hilbert space. The latter property leads to a possible quantum avoidance of the singularity ("cosmological bounce"), and suggests imposing the boundary condition that the wavefunction of the Universe vanish when the volume of space tends to zero (a type of boundary condition which looks like a final-state condition when considering the big crunch inside a black hole). The space of solutions is a mixture of "discretespectrum states" (parametrized by a few constant parameters, and known in explicit form) and of continuous-spectrum states (parametrized by arbitrary functions entering some initial-value problem). The predominantly negative values of the squared-mass term lead to a "bottle effect" between small-volume-Universes and large-volume ones, and to a possible reduction of the continuous spectrum to a discrete spectrum of quantum states looking like excited versions of the Planckian-size Universes described by the discrete states at fermionic levels $N_{F}=0$ and 1.

The interested reader will find all the details in references [1,2].

\section{References}

[1] T. Damour, Ph. Spindel, Quantum supersymmetric cosmology and its hidden Kac-Moody structure, Class. Quant. Grav. 30 (2013), 162001; arXiv: 1304.6381 [gr-qc]

[2] T. Damour, Ph. Spindel, Quantum supersymmetric Bianchi IX cosmology, Phys. Rev. D 90 (2014), 103509; arXiv: 1406.1309 [gr-qc] 